\section{Personalización en aprendizaje colaborativo soportado por computadora mediante sistemas basados en agentes}

Recibido: 1 Julio 2015 - Revisado: 30 Septiembre 2015

Aceptado: 30 Octubre 2015 - Publicado: 30 Diciembre 2015

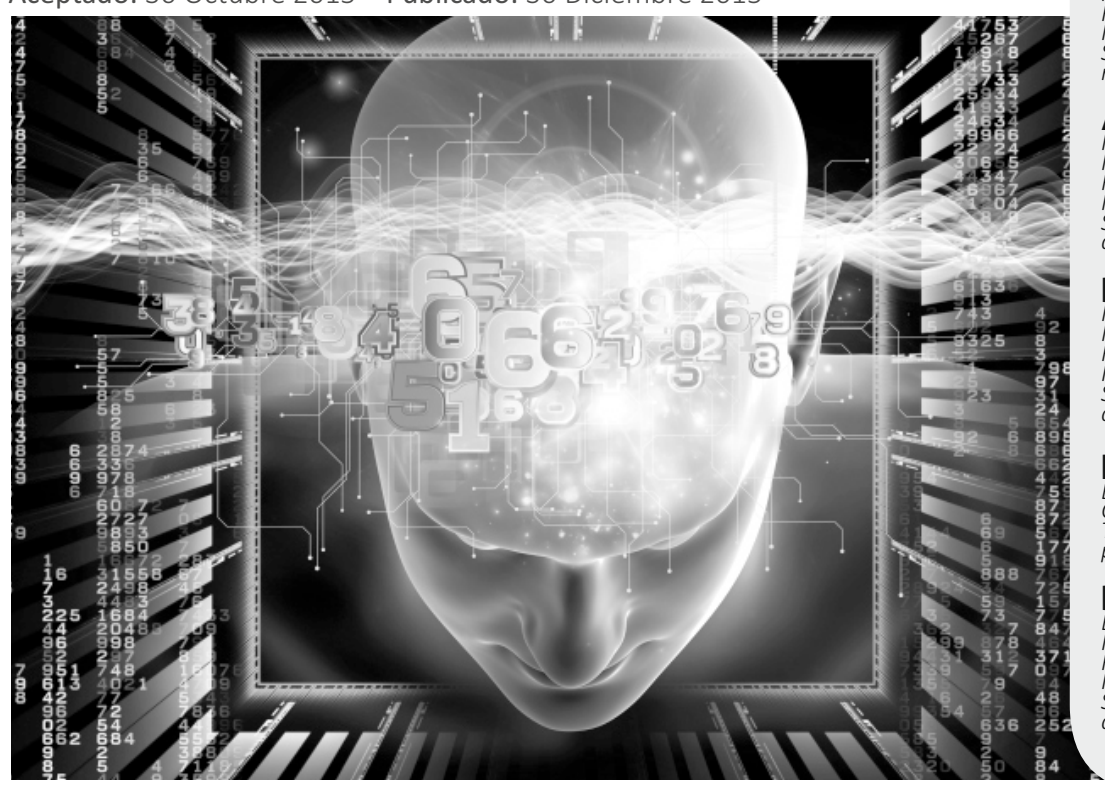

Rosanna Costaguta

Investigador Director de Proyecto, Instituto de

Investigacion en Informática y Sistemas de Información, Facultad de Ciencias Exactas y Tecnologías, Universidad Nacional de Santiago del Estero, Av. Belgrano (S) 1912, Santiago del Estero, Argentina. rosanna@unse.edu.ar

\section{María de los Angeles Menini}

Investigador Integrante de Proyecto, Instituto de Investigación en Informática y Sistemas de Información, Facultad de Ciencias Exactas y Tecnologías, Universidad Nacional de Santiago del Estero, Av. Belgrano (S) 1912, Santiago del Estero, Argentin marameni@unse.edu.ar

\section{Aldo Roldán}

nvestigador Integrante de Proyecto, Instituto de Investigacion en Informática y Sistemas de Información, Facultad de Ciencias Exactas y Tecnologias, Universidad Nacional de Santiago del Estero, Av. Belgrano (S) 1912, Santiago del Estero, Argentin aroldan@unse.edu.ar

\section{Daniela Missio}

Investigador Integrante de Proyecto, Instituto de Investigación en Informática y Sistemas de Información, Facultad de Ciencias Exactas y Tecnologias, Universidad Nacional de Santiago del Estero, Av. Belgrano (S) 1912. Santiago del Estero, Argentin dmissio@unse.edu.ar

\section{Pablo Santana-Mansilla}

Becario doctoral Integrante de Proyecto, CONICET Consejo Nacional de Investigaciones Cientificas y psantana@unseina.

\section{Diego Yanacon Atia}

Estudiante Integrante de Proyecto, Instituto de Investigación en Informática y Sistemas de Información, Facultad de Ciencias Exactas y Tecnologías, Universidad Nacional de Santiago del Estero, Av. Belgrano (S) 1912, diegopunk27@hot, Argentina.

Resumen: En los sistemas de Aprendizaje Colaborativo Soportado por Computadoras no alcanza con organizar a los estudiantes en grupos. El éxito en las experiencias de colaboración está condicionado (entre otros factores) por los roles que tanto estudiantes como profesores sean capaces de manifestar durante la dinámica de trabajo. En este artículo se presentan dos sistemas basados en agentes especialmente diseñados. El primero está enfocado tanto a monitorear los roles de equipo desempeñados por los estudiantes en la dinámica de colaboración, como a intervenir de manera automática con acciones correctivas cuando esos roles no se muestran adecuadamente. El segundo sistema basado en agentes apunta tanto a la detección de conflictos grupales que requieran la intervención del profesor para resolverse, como a llevar a cabo el entrenamiento de esos profesores en la manifestación de los roles adecuados para resolver esos conflictos. Ambos sistemas están actualmente en desarrollo para su posterior validación experimental con estudiantes y docentes reales.

Palabras clave: Aprendizaje Colaborativo Soportado por Computadoras, roles de equipo, e-tutores, sistema basado en agentes, roles de e-tutores.

Abstract: In systems for Computer Supported Collaborative Learning is not enough to organize students into groups. The successful in a collaborative experience depends on (among other things) the roles that students and teachers are able to manifest during the collaborative dynamic. In this paper two agent-based systems specially designed are presented. The first is focused both monitoring team roles played by students in the dynamics of collaboration, such as automatically intervene with corrective action when these roles are not properly displayed. The second agent-based system aimed at detecting group conflicts that require teacher intervention to resolve, and to carry out the training of those teachers in the demonstration of the proper roles to resolve these conflicts. Both systems are currently under development for further experimental validation with real students and teachers.

Key words: Computer Supported Collaborative Learning; team roles; e-tutor; agent-based systems; e-tutor roles. 


\section{INTRODUCCIÓN}

Un grupo es un conjunto dinámico de personas que trabajan juntas para alcanzar un objetivo común (Delgado et al., 2001). Cuando ese trabajo se realiza utilizando la computadora como medio de comunicación y de colaboración, en un entorno de enseñanza y de aprendizaje, se está en presencia de grupos de Aprendizaje Colaborativo Soportado por Computadoras (ACSC).

El ACSC fue rápidamente adoptado en el ámbito de la enseñanza a distancia. Esto se debe fundamentalmente a que, entre otras ventajas, permite a los integrantes de cualquier grupo independizarse de las variables tiempo y espacio. Así, estudiantes ubicados en puntos geográficos distantes pueden trabajar colaborativamente, incluso contribuyendo en momentos distintos en el tiempo. A pesar de esto, crear grupos e instar a sus miembros a resolver una consigna de manera colaborativa no garantiza en forma alguna que el comportamiento y el rendimiento de esos grupos sean los adecuados, ni que la experiencia de enseñanza y de aprendizaje sea exitosa. La colaboración conduce a resultados positivos cuando los integrantes del grupo dan y reciben ayuda, comparten recursos, negocian con sus pares, construyen conocimientos, adquieren competencias, resuelven conflictos y se regulan mutuamente manifestando habilidades interpersonales.

En un grupo colaborativo, los estudiantes trabajan juntos para alcanzar eventualmente alguna meta prefijada (Onrubia y Engel, 2012); cada uno de ellos es responsable por sus acciones, pero a la vez, todos trabajan juntos sobre el mismo problema y respetan las habilidades y contribuciones de cada uno. En este tipo de grupo es fundamental que los integrantes desempeñen diferentes funciones o roles que permitan realizar una labor coordinada. Así, es indudable que el éxito o fracaso de una experiencia de ACSC depende en gran medida de los roles que sean capaces de manifestar los miembros del grupo. Ante esta situación, es necesario desarrollar técnicas orientadas a mejorar el desempeño de los estudiantes dentro de los grupos de ACSC a través de la adecuada manifestación de los roles.

Por oro lado, en ACSC los integrantes de un grupo no son solo los estudiantes, muchas veces la oportuna intervención del docente o e-tutor resulta imprescindible. Un e-tutor en ACSC es quien apoya los procesos de enseñanza y de aprendizaje por medio de la interacción directa con los estudiantes (Sulčič y Sulčič, 2007). Por ello, le corresponde al e-tutor de ACSC, estructurar y guiar los procesos colaborativos de los estudiantes, con el fin de generar las interacciones que propicien la construcción colaborativa de conocimiento (Onrubia y Engel, 2012).

$\mathrm{Si}$ se tiene en cuenta que los e-tutores de ACSC usualmente no saben cuál es la manera más efectiva de asistir a los estudiantes (Kukulska-Hulme, 2004), que además no pueden seguir utilizando las técnicas propias del aula tradicional con las cuales están familiarizados (Orvis y Lassiter, 2006) y que existen pocas chances de éxito sin docentes con las habilidades necesarias para sacarle provecho a las herramientas tecnológicas disponibles (Hughes et al., 2002; Kukulska-Hulme, 2004; Orvis y Lassiter, 2006), es evidente que los e-tutores requieren capacitación y formación. Ante esta particularidad, es indispensable plantear mecanismos que permitan a los e-tutores de ACSC, adquirir las habilidades necesarias para desempeñarse adecuadamente.

Dada la realidad presentada en los párrafos previos, se identifican dos situaciones conflictivas. Por un lado, grupos de ACSC no mediados por docentes, donde los estudiantes no manifiestan adecuadamente los roles necesarios para desarrollar exitosamente la experiencia. Por otro lado, otros grupos de ACSC con mediación docente, donde los e-tutores tampoco desempeñan adecuadamente sus roles como tales. 
En este trabajo se plantean dos sistemas basados en agentes diseñados para dar solución a las problemáticas expuestas.

Este artículo se organiza como se explica a continuación. La próxima sección describe primero al sistema basado en agentes aplicable a grupos de ACSC que no cuentan con el apoyo de un e-tutor y luego, presenta el segundo sistema basado en agentes destinado a grupos de ACSC guiados por un e-tutor. La sección 3 enuncia antecedentes vinculados con los sistemas descriptos en la sección 2. Finalmente, la sección 4 contiene algunas conclusiones.

\section{SISTEMAS PROPUESTOS}

En esta sección se describen los dos sistemas basados en agentes ideados para solucionar las problemáticas planteadas en la sección previa. El primer sistema hace hincapié en el comportamiento de los estudiantes dentro de un grupo de ACSC, mientras que el segundo apunta al accionar de los e-tutores en experiencias de ACSC. Cabe destacar que ambos sistemas basados en agentes se encuadran en la categoría más alta planteada por Soller et al. (2005), es decir, los sistemas de ACSC que guían o aconsejan.

\subsection{Monitoreo de roles de estudiantes}

En un grupo de ACSC, sus integrantes se involucran, desde su saber, trabajan juntos para alcanzar un objetivo común, la construcción del conocimiento. En estos grupos, los estudiantes se relacionan y expresan conductas, de tal modo que pueden establecer por la forma de comportarse, un cierto y determinado rol.

Un rol se define como la tendencia a comportarse, contribuir e interrelacionarse de una determinada manera con el resto de los integrantes de un equipo (Belbin, 1996). Si bien las conductas o roles de un individuo en un equipo pueden ser infinitas, el rango de conductas útiles que realizan una contribución efectiva al equipo es finito (Belbin, 2001).

Una de las clasificaciones de roles más utilizadas es la propuesta por Belbin (1996), quien afirma que existen nueve roles diferentes que al ser manifestados por los integrantes de un grupo, impactan en la efectividad del trabajo. Cuando un equipo puede equilibrar la aparición de estos roles, el alcanzar con éxito un trabajo coordinado está asegurado (Belbin, 2001). Dichos roles son: (a) Impulsor, (b) Implementador, (c) Finalizador, (d) Coordinador, (e) Cohesionador, (f) Investigador de Recursos, (g) Cerebro, (h) Monitor-Evaluador y (i) Especialista (Belbin, 1996). Cada uno de ellos presenta características distintivas (Tabla 1).

Por ejemplo, un estudiante desempeñando el rol Cerebro podría tratar de imponer sus ideas, lo que entorpecería la dinámica de colaboración. Un estudiante Impulsor que continuamente descalifica las contribuciones de sus compañeros, o un estudiante Implementador que inflexiblemente rechaza las nuevas ideas o propuestas de solución de sus compañeros son claros ejemplos de roles que no se están desenvolviendo adecuadamente.

Tabla 1. Roles de Belbin.

\begin{tabular}{|c|c|c|}
\hline Rol & Descripción. & Puntos débiles. \\
\hline Cerebro & Creativo, imaginativo, poco ortodoxo. Resuelve \\
problemas difíciles. & $\begin{array}{c}\text { Suele dispersarse o distraerse. } \\
\text { Trata de imponerse }\end{array}$ \\
\hline Coordinador & Maduro, seguro de sí mismo. Aclara las metas a \\
& alcanzar. Promueve la toma de decisiones. Delega bien. & $\begin{array}{c}\text { Suele delegar demasiado en sus } \\
\text { compañeros de equipo. }\end{array}$ \\
\hline
\end{tabular}

Rosanna Costaguta, María de los Angeles Menini, Aldo Roldán, Daniela Missio, Pablo Santana-Mansilla y Diego Yanacon Atia: Personalización en aprendizaje colaborativo soportado por computadora mediante sistemas basados en agentes. Revista TEKNOS, 15 (2) Pag. 9- 17 


\begin{tabular}{|c|c|c|}
\hline Rol & Descripción. & Puntos débiles. \\
\hline Monitor-Evaluador & $\begin{array}{c}\text { Serio, perspicaz y estratega. Percibe todas las opciones. } \\
\text { Juzga con exactitud. }\end{array}$ & $\begin{array}{l}\text { Carece de habilidad para } \\
\text { motivar a otros. }\end{array}$ \\
\hline Implementador & $\begin{array}{l}\text { Disciplinado, leal, conservador y eficiente. Transforma } \\
\text { las ideas en acciones. }\end{array}$ & $\begin{array}{l}\text { Suele ser poco flexible y resistirse } \\
\text { a nuevas ideas. }\end{array}$ \\
\hline Finalizador & $\begin{array}{l}\text { Esmerado, concienzudo, ansioso. Busca los errores y } \\
\text { las omisiones. Realiza las tareas en el plazo establecido. }\end{array}$ & $\begin{array}{l}\text { Tiende a concentrarse demasiado } \\
\text { en detalles. }\end{array}$ \\
\hline $\begin{array}{l}\text { Investigador de } \\
\text { Recursos }\end{array}$ & $\begin{array}{c}\text { Extrovertido, entusiasta, comunicativo. Busca nuevas } \\
\text { oportunidades. Desarrolla contactos. }\end{array}$ & $\begin{array}{l}\text { Tiende a perder en interés inicial } \\
\text { desmotiván dose en el proceso. }\end{array}$ \\
\hline Impulsor & $\begin{array}{l}\text { Retador, dinámico, trabaja bien bajo presión. Tiene } \\
\text { iniciativa y coraje para superar los obstáculos. }\end{array}$ & $\begin{array}{l}\text { Tiende a impacientarse y } \\
\text { descalificar a otros. }\end{array}$ \\
\hline Cohesionador & $\begin{array}{l}\text { Cooperador, apacible, perceptivo y diplomático } \\
\text { Escucha e impide los enfrentamientos.. }\end{array}$ & $\begin{array}{l}\text { Suelen tornarse indecisos en } \\
\text { los momentos cruciales. }\end{array}$ \\
\hline Especialista & $\begin{array}{l}\text { Sólo le interesa una cosa a un tiempo. Aporta } \\
\text { cualidades y conocimientos específicos. }\end{array}$ & $\begin{array}{l}\text { Suelen contribuir solo cuando } \\
\text { se tratan temas específicos. }\end{array}$ \\
\hline
\end{tabular}

De lo anterior, se desprende la necesidad de identificar los roles que efectivamente manifiesta cada uno de los integrantes de un grupo de ACSC, y diagnosticar el estado actual de la colaboración con el fin de intervenir en el momento adecuado y de ese modo, propiciar alcanzar un equilibrio de roles y un ACSC exitoso.

Así, el primer sistema basado en agentes tiene por objetivos: (a) identificar los roles que manifiestan los estudiantes mientras trabajan en grupo, (b) diagnosticar el estado de la colaboración e (c) intervenir con acciones correctivas cuando los roles no se muestren adecuadamente.

La estructura elegida para modelar el diálogo grupal es el árbol de discusión, donde la consigna original (dada por el docente) constituye el nodo raíz a partir del cual se incorporarán ramas a medida que los estudiantes propongan nuevas ideas o discutan las expuestas. Para dialogar, los estudiantes utilizarán oraciones de apertura, es decir, seleccionarán desde la interfaz la frase con la cual desean comenzar su contribución, para luego, continuar escribiendo texto libre (Soller, 2001). Para definir el conjunto de oraciones de apertura, se considerará una investigación previa realizada por autores de este trabajo (Costaguta et al., 2011).

Durante la experimentación, todas las interacciones que manifiesten los estudiantes serán almacenadas y un agente de interfaz las analizará para actualizar el modelo de usuario o perfil de colaboración de cada estudiante. Otro agente, considerando esos perfiles, diagnosticará el estado de la colaboración y determinará si es necesario realizar alguna intervención con la finalidad que los roles de equipo se manifiesten adecuadamente. Este agente contará con una red bayesiana diseñada especialmente para reconocer necesidades de intervención. Las acciones 
correctivas a implementar por este agente se definirán con el asesoramiento de un psicopedagogo.

\subsection{Monitoreo de roles de e-Tutores}

La coordinación de discusiones supone para los etutores de ACSC, una considerable carga tanto temporal como cognitiva. En la última década, se desarrollaron aplicaciones de software que proporcionan a los e-tutores, información clave del proceso de interacción para que puedan monitorear, evaluar y guiar a los grupos de estudiantes hacia discusiones fructíferas (Schwarz y Asterhan, 2011) (Tchounikine et al., 2010). En este tipo de aplicaciones, el análisis de las interacciones es fundamental, porque permite conocer lo que hacen los estudiantes cuando se comunican con sus pares.

En los ambientes de ACSC, las interacciones que son ideales para el aprendizaje no ocurren espontáneamente (Chen, 2006; Costaguta et al., 2011; Godoy et al., 2004) y el uso de computadoras incluso puede llegar a inhibirlas (Hughes et al., 2002), por lo que resulta imprescindible la oportuna intervención del e-tutor. Si se tiene en cuenta que los e-tutores de ACSC usualmente no saben cuál es la manera más efectiva de asistir a los estudiantes, que además no pueden seguir utilizando las técnicas propias del aula tradicional con las cuales están familiarizados y que existen pocas chances de éxito sin docentes con las habilidades necesarias para sacarle provecho a las herramientas tecnológicas disponibles (Hughes et al., 2002; Krippendorff, 2004); es evidente que los e-tutores requieren capacitación y formación.

Hasta el momento, para modelar las interacciones se han utilizado tanto interfaces no estructuradas (texto libre) como interfaces estructuradas o semiestructuradas (oraciones de apertura, contribuciones dirigidas por menú y actos de diálogo). Mientras el texto libre no condiciona el contenido de las interacciones, al mismo tiempo dificulta el análisis, puesto que requiere aplicar técnicas de procesamiento de lenguaje natural. Por el contario, con las interfaces estructuradas o semiestructuradas, se simplifica el proceso de análisis de las contribuciones (Soller et al., 2005; Tchounikine et al., 2010); no obstante, esta simplificación puede conducir a interpretaciones erróneas (Tchounikine et al., 2010) y hasta afectar negativamente la colaboración al restringir los tipos de actos comunicativos (Chen, 2006; Soller et al., 2005).

Por otro lado, es necesario considerar que los docentes usualmente no poseen las habilidades requeridas para desenvolverse adecuadamente en entornos de ACSC. Dichas habilidades pertenecen a la categoría de habilidades adquiridas, por cuanto no son habilidades que una persona posea como consecuencia de su existencia, sino que pueden desarrollarse mediante procesos de aprendizaje y entrenamiento (Barker, 2010).

Ante esta situación, es necesario plantear mecanismos que permitan a los e-tutores de ACSC, adquirir las habilidades necesarias para desempeñarse adecuadamente.

Así, el segundo sistema basado en agentes tiene por objetivos diagnosticar el estado de la colaboración, identificar los conflictos que se produzcan mientras los estudiantes trabajan en grupo y definir mecanismos de recomendación personalizados a fin de sugerir a los e-tutores, las acciones adecuadas para resolver tales conflictos.

El diálogo entre estudiantes e e-tutores se realizará con base en texto libre, por lo cual se aplicarán técnicas de minería de datos y de procesamiento de lenguaje natural para analizar las interacciones. Para reconocer situaciones problemáticas en la dinámica de trabajo grupal, se están evaluando los siguientes métodos: IPA (Bales, 1950), Symlog (Bales, 1979) y el modelo de toma de decisión en grupos de Poole (Poole y Roth, 1989). Para categorizar las intervenciones de los e-tutores, se considerará la taxonomía de habilidades propuesta por Santana- 
Mansilla et al. (2011). Con la ayuda de psicopedagogos con experiencia en ACSC, se establecerá una vinculación entre la taxonomía y las situaciones problemáticas grupales y se definirán las intervenciones correctivas adecuadas que se espera manifiesten los e-tutores en esos casos.

Durante la experimentación todas las interacciones serán almacenadas, un agente de interfaz las analizará con el fin de diagnosticar el estado de la colaboración y reconocer la existencia de conflictos. Cuando esto ocurra, otros agentes sugerirán a los e-tutores las acciones a llevar a cabo para resolver esos conflictos y mejorar el aprendizaje grupal.

\section{ANTECEDENTES}

Considerando el sistema basado en agentes propuestos para monitorear los roles de los estudiantes, se toman como antecedentes los siguientes trabajos. Strijbos et al. (2005) documentan dos experiencias llevadas a cabo para determinar roles que aparecen en ACSC, distingue entre roles funcionales y no funcionales. Los autores concluyen en que existe un mejor desempeño en grupos donde se destacan los roles funcionales.

Schellens et al. (2005) discuten la influencia de las características de los estudiantes, del grupo y también de las tareas, sobre el nivel de construcción de conocimiento alcanzado por grupos de ACSC. Los autores demuestran mayor influencia respecto a las características de las tareas a realizar; los roles son una de las características consideradas.

Liao et al. (2008) aplican técnicas de minería de datos (clustering) para analizar las interacciones registradas en sesiones de ACSC y descubrir roles dominantes.

Considerando el sistema basado en agentes propuesto para monitorear los roles de los e-tutores, se toman como antecedentes, algunas investigaciones donde sus aplicaciones de software proporcionan a los e-tutores (de manera resumida), información sobre aspectos clave del proceso de interacción para que puedan monitorear, evaluar y guiar a los conjuntos de estudiantes hacia discusiones fructíferas (Schwarz y Asterhan, 2011; Tchounikine et al., 2010).

En particular, el material encontrado sobre las habilidades de e-tutores en ACSC es muy escaso. Sin embargo, entre los investigadores que han estudiado esta temática, se pueden identificar dos enfoques. Por un lado, se proporcionan guías y recomendaciones para e-tutores de ACSC, pero sin brindar mayores precisiones. Por otra parte, se analiza el rol de los etutores de ACSC tomando como base el esquema de organización por roles, heredado de la literatura sobre habilidades y competencias para docentes de elearning, que no recurren al aprendizaje colaborativo como estrategia pedagógica, sino que se basan en el modelo de interacción docente-estudiante.

No obstante, no se puede hacer una transferencia directa de las habilidades planteadas, puesto que en la dinámica grupal surgen problemas que no están presentes cuando los estudiantes aprenden de manera individual (estudiantes que nunca participan, estudiantes que tienden a dominar el diálogo, discusiones $\sin$ fin, etc.).

Así, el sistema basado en agentes que se va a desarrollar tomará como antecedente válido un esquema de clasificación de las habilidades que se considera debe poseer un docente para desempeñarse de manera efectiva en entornos de ACSC. Esta taxonomía elaborada por parte de los autores de este artículo (Santana-Mansilla et al, 2011) se organiza en tres niveles consecutivos de detalle, donde cada nivel proporciona más particularidades de su predecesor.

El primer nivel considera las habilidades vinculadas con los roles que desempeñan los e-tutores al 
coordinar el aprendizaje: Administrativo, Pedagógico, Social, Técnico, Comunicación y Evaluación. El segundo nivel especifica las subhabilidades relacionados con cada habilidad y finalmente, el tercer nivel describe cada subhabilidad en términos de atributos de habilidad.

Además, existen seis sistemas de ACSC que podrían vincularse particularmente con los sistemas propuestos, dado que aconsejan acciones en función del análisis de las interacciones registradas. Ellos son: DEGREE (Barros y Verdejo, 2000), aLFanet (Santos et al., 2003), COLER (Constantino-González et al. 2003; Chen, 2006), ECOLA (Suh y Lee, 2006), I-MINDS (Soh et al., 2006) y SAVER (Costaguta et al., 2011). No obstante, en ninguno de ellos se tienen en cuenta los roles de equipo ni las habilidades de los e-tutores. Adicional a esto, no se hallaron antecedentes vinculados con la utilización de técnicas de Inteligencia Artificial para propiciar la adecuada manifestación de roles en grupos de ACSC, ni para entrenar de manera personalizada las habilidades de los e-tutores en grupos de ACSC, todo lo cual destaca la originalidad de estas propuestas.

\section{CONCLUSIONES}

Esta investigación se orienta a generar conocimiento científico-tecnológico de relevancia para el desarrollo de sistemas de información web personalizados en el área del ACSC. Asimismo, realiza propuestas metodológicas y desarrollos de módulos para sistemas de información web en ACSC (mediante la inclusión de modelos de usuario, agentes y técnicas de aprendizaje de máquina). En particular, la investigación que se describió a lo largo de este artículo encara dos enfoques diferentes tendientes a potenciar los resultados de las experiencias colaborativas de aprendizaje online. Cada uno de esos enfoques constituye una de las líneas de trabajo que se están ejecutando actualmente dentro del proyecto de investigación «Sistemas de información web basados en agentes para promover el Aprendizaje Colaborativo Soportado por Computadoras» (CICYT UNSE Código 23/C097), para el período 2012-2016.

Es indudable que investigar en ACSC implica el análisis de complejas relaciones entre diversas variables y actores involucrados. Para que un grupo de ACSC trabaje de manera coordinada y eficiente, los roles que los estudiantes y los e-tutores sean capaces de desempeñar resultan determinantes. Se cree que el primer sistema basado en agentes propuesto contribuirá a incrementar los mecanismos disponibles para monitoreo de grupos; esto resulta innovador en tanto considera, para ello, los roles de equipo que manifiesten los miembros. También, se considera innovador al segundo sistema propuesto basado en agentes, puesto que mediante un entrenamiento personalizado, permitirá dar un paso importante en la capacitación de los e-tutores de ACSC.

Actualmente, ambos sistemas para monitoreo de roles de estudiantes y de e-tutores están en desarrollo, previéndose validar sus respectivos funcionamientos mediante experiencias reales de ACSC que se llevarán a cabo durante el primer semestre de 2016 con estudiantes y docentes universitarios. 


\section{REFERENCIAS}

Bales, R., Cohen, S., y Williamson, S. (1979). Symlog: A system for the multiple level observation of groups. The Free Press.

Bales, R. (1950). A set of categories for the analysis of small group interaction. American Sociological Review, 15(2), 257-263.

Belbin, M. (2001). Managing without Power. Oxford: Butterworth-Heinemann.

Barker, P. (2010). Introduction. Electronic Performance Support Using Digital Technology to Enhance Human Ability. Inglaterra: Gower.

Barros, B. y Verdejo, M. (2000). Analysing student interaction processes in order to improve collaboration. The DEGREE approach. International Journal of Artificial Intelligence in Education, 11, 221-41.

Belbin, M. (1996). Team Roles at Work (2nd Ed.). Oxford: Butterworth-Heinemann.

Chen, W. (2006). Supporting teachers' intervention in collaborative Knowledge building. Journal of Network and Computer Applications, 29, 200-215.

Constantino-González, M., Suthers, D., y Escamilla de los Santos, J. (2003). Coaching Web-based Collaborative Learning based on Problem Solution Differences and Participation. International Journal of Artificial Intelligence in Education, 13, 263-299.

Costaguta, R., Amandi, A., y García, P. (2011). Using Agents for Training Students Collaborative Skills. IEEE Latin America Transactions, 9(7), 118-1124.

Delgado, A., Olguín, C., y Ricarte, I. (2001). Monitoring Learners Activities in a Collaborative Environment. Proc. 7th IEEE International Workshop on Groupware. Alemania.

Godoy, D., Schiaffino, S., y Amandi, A. (2004). Interface agents personalizing Web-based tasks. Special Issue on Intelligent Agents and Data Mining for Cognitive Systems, Cognitive Systems Research Journal, 5 (3), 207-222.
Hughes, S., Wickersham, L., Ryan-Jones, D., y Smith, S. (2002). Overcoming Social and Psychological Barriers to Effective On-line Collaboration. Educational Technology \& Society, 5(1), 86-92.

Kukulska-Hulme, A. (2004). Do Online Collaborative Groups Need Leaders? Online Collaborative Learning: Theory and Practice, Information Science Publishing, 262-280.

Krippendorff, K. (2004). Content analysis: an introduction to its methodology., Filadelfia: SAGE Publications.

Liao, J., Li, Y., Chen, P., y Huang, R. (2008). Using Data Mining as Strategy for Discovering User Roles in CSCL. 8th IEEE Internatinal Conference on Advanced Learning Technologies. España.

Onrubia, J., y Engel, A. (2012). The role of teacher assistance on the effects of a macro-script in collaborative writing tasks. International Journal of Computer Supported Collaborative Learning, 7(1), 161-186.

Orvis, K. y Lassiter, A (2006). Computer-Supported Collaborative Learning: The Role of the Instructor. Teaching and learning with virtual teams, Information Science Publishing, 158-179.

Poole, M., y Roth, J. (1989). Decision Development in Small Groups V: Test of a Contingency Model. Human Communication Research, 15(4), 549-589.

Santana-Mansilla, P., Costaguta, R., y Missio, D. (2011). Habilidades de e-tutores en grupos colaborativos. Proc. IV Simp. Int. de Investigación, La investigación Aplicada en la Universidad: Experiencias innovadoras en investigación aplicada. Argentina: Jujuy.

Santos, O., Barrera, C., Gaudioso, E., y Boticario, J. (2003). ALFANET: An adaptive e-learning platform. Proc. 2nd International Meeting on Multimedia and Information and Communication Technologies in Education, 1938-1942.

Schellens, T, Van Keer, H., Valcke, M., y De Wever, B. (2005). The Impact of role Assignment as Scripting Tool on Knowledge Construction in Asynchronous Discussion Groups. ACM Conference on Computer Support for Collaborative Learning. Taiwan. 
Schwarz, B., y Asterhan, C. (2011). E-Moderation of Synchronous Discussions in Educational Settings: A Nascent Practice. Journal of the Learning Sciences, 20(3), 395-442.

Soh, L., Khandaker, N., y Jiang, H. (2006). I-MINDS: A Multiagent System for Intelligent Computer-Supported Collaborative Learning and Classroom Management. International Journal of Artificial Intelligence in Education, $28(2), 119-151$.

Soller, A. (2001). Supporting Social Interaction in an Intelligent Collaborative Learning System. International Journal of Artificial Intelligence in Education, 12, 40-62.

Soller, A., Martínez, M.., Jermann, P., \& Muehlenbrock, M. (2005). From Mirroring to Guiding: A Review of State of the Art Technology for Supporting Collaborative Learning. International Journal of Artificial Intelligence in Education, 15(4), 261-290.

Strijbos, J., de Laat, M., Martens, R., y Jochems, W. (2005). Functional versus Spontaneous Roles during CSCL. ACM Conference on Computer Support for Collaborative Learning. Taiwan.

Suh, H., y Lee, S. (2006). Collaborative Learning Agent for Promoting Group Interaction. ETRI Journal, 28(4), 461-474. Sulčič, V., y Sulčič, A. (2007). Can Online Tutors Improve the Quality of E-Learning?. Issues in Informing Science and Information Technology, 4, 210-210.

Tchounikine, P., Rummel, N., y McLaren, B. (2010). Computer Supported Collaborative Learning and Intelligent Tutoring Systems. Advances in Intelligent Tutoring Systems, Springer, 447-463. 